\title{
MEAN SQUARE FORMULA FOR THE DOUBLE ZETA-FUNCTION
}

\author{
Isao Kivchi, Makoto Minamide
}

\begin{abstract}
We prove the mean square formula of the Euler-Zagier type double zeta-function $\zeta_{2}\left(s_{1}, s_{2}\right)$ and provide an improvement on the $\Omega$ results of Kiuchi, Tanigawa, and Zhai. We also calculate the double integral $\int_{2}^{N} \int_{2}^{T}\left|\zeta_{2}\left(s_{1}, s_{2}\right)\right|^{2} d t_{1} d t_{2}$ under certain conditions.
\end{abstract}

Keywords: double zeta-functions, mean square formula, Riemann zeta-function.

\section{Introduction}

Let $s_{j}=\sigma_{j}+i t_{j}(j=1,2)$ be complex variables with $\sigma_{j}, t_{j} \in \mathbb{R}$, and let $\zeta(s)$ be the Riemann zeta-function, which is defined as $\zeta(s):=\sum_{n=1}^{\infty} n^{-s}$ for $\operatorname{Re} s>1$. The double zeta-function of Euler-Zagier type is defined by

$$
\zeta_{2}\left(s_{1}, s_{2}\right)=\sum_{1 \leqslant m<n} \frac{1}{m^{s_{1}} n^{s_{2}}},
$$

which is absolutely convergent for $\sigma_{2}>1$ and $\sigma_{1}+\sigma_{2}>2$. The proof of Atkinson's formula for the mean value theorem of the Riemann zeta-function $\zeta(s)$ (see F.V. Atkinson [2] or A. Ivić [6]) applies to the function (1.1). Some analytic properties of (1.1) have been obtained by S. Akiyama, S. Egami and Y. Tanigawa [1], H. Ishikawa and K. Matsumoto [5], I. Kiuchi and Y. Tanigawa [9], I. Kiuchi, Y. Tanigawa and W. Zhai [10], K. Matsumoto [11], [12], J. Q. Zhao [15], and others.

K. Matsumoto and H. Tsumura [13] were the first to study a new type of mean value formula for $\int_{2}^{T}\left|\zeta_{2}\left(s_{1}, s_{2}\right)\right|^{2} d t_{2}$ with a fixed complex number $s_{1}$ and any number $T>2$. They conjectured that when $\sigma_{1}+\sigma_{2}=\frac{3}{2}$, the form of the main term of the mean square formula would not be $C T$ with a constant $C$, and that, most probably, some log-factor would appear. Recently, their results were 
considered by S. Ikeda, K. Matsuoka and Y. Nagata [4], who showed that

$$
\begin{aligned}
\int_{2}^{T}\left|\zeta_{2}\left(s_{1}, s_{2}\right)\right|^{2} d t_{1}= & \left(\sum_{m=1}^{\infty} \frac{1}{m^{2 \sigma_{1}}}\left|\zeta\left(s_{2}\right)-\sum_{n=1}^{m} \frac{1}{n^{s_{2}}}\right|^{2}\right) T \\
& +\left\{\begin{array}{cl}
O\left(T^{4-2 \sigma_{1}-2 \sigma_{2}}\right) & \text { if } \frac{3}{2}<\sigma_{1}+\sigma_{2}<2, \\
O\left(\log ^{2} T\right) & \text { if } \sigma_{1}+\sigma_{2}=2 .
\end{array}\right.
\end{aligned}
$$

Here, the coefficient of the main term on the right-hand side of (1.2) converges if $\sigma_{1}+\sigma_{2}>\frac{3}{2}$. They also deduced that the asymptotic formula

$$
\int_{2}^{T}\left|\zeta_{2}\left(s_{1}, s_{2}\right)\right|^{2} d t_{1}=\frac{1}{\left|s_{2}-1\right|^{2}} T \log T+O(T)
$$

holds on the line $\sigma_{1}+\sigma_{2}=\frac{3}{2}$. This result implied that the conjecture of Matsumoto and Tsumura on the line $\sigma_{1}+\sigma_{2}=\frac{3}{2}$ was true.

Before the introduction of our theorems, let us recall the mean square formulas of the Riemann zeta-function $\zeta(\sigma+i t)$ (see [2], [6], [7], [14]). It is well known that

$$
\int_{2}^{T}|\zeta(\sigma+i t)|^{2} d t=\zeta(2 \sigma) T+O(1)
$$

with $\sigma>1$, and

$$
\int_{2}^{T}\left|\zeta\left(\frac{1}{2}+i t\right)\right|^{2} d t=T \log \frac{T}{2 \pi}+(2 \gamma-1) T+O\left(T^{\frac{1}{2}}\right),
$$

where $\gamma$ denotes the Euler constant. For $\frac{1}{2}<\sigma<1$, A. Ivić and K. Matsumoto [8] obtained that

$$
\int_{2}^{T}|\zeta(\sigma+i t)|^{2} d t=\zeta(2 \sigma) T+A(\sigma) T^{2-2 \sigma}+E_{\sigma}(T)
$$

with $A(\sigma)=(2 \pi)^{2 \sigma-1} \frac{\zeta(2-2 \sigma)}{2-2 \sigma}$, where the error term of (1.6) was estimated as $E_{\sigma}(T)=O\left(T^{\frac{2}{3}(1-\sigma)} \log ^{2 / 9} T\right)$. The mean square formula of $\zeta(1+i t)$ was obtained by R. Balasubramanian, A. Ivić, and K. Ramachandra [3], who showed that

$$
\int_{2}^{T}|\zeta(1+i t)|^{2} d t=\zeta(2) T-\pi \log T+R(T)
$$

with $R(T)=O\left((\log T)^{2 / 3}(\log \log T)^{1 / 3}\right)$, and the estimate

$$
\int_{2}^{T} R(t) d t=O(T)
$$


The main purpose of this paper is to prove the mean square formula for the double zeta-function $\zeta_{2}\left(s_{1}, s_{2}\right)$ within the region $0<\sigma_{1}<1,0<\sigma_{2}<1$ and $0<\sigma_{1}+\sigma_{2}<\frac{3}{2}$. Ikeda, Matsuoka, and Nagata made use of the mean value theorems for Dirichlet polynomials and suitable approximations to the Euler-Maclaurin summation formula to obtain the formulas (1.2) and (1.3). We use the formulas (1.4)-(1.8) for the mean square of $|\zeta(\sigma+i t)|$ and $(2.8)-(2.10)$ in Lemma 3 below, as well as Lemma 1 below, which was derived from a weak form of the approximate formula of Kiuchi, Tanigawa, and Zhai [10] for $\zeta_{2}\left(s_{1}, s_{2}\right)$ to obtain the following formula.

Theorem 1. Suppose that $2 \leqslant t_{1} \leqslant T, \quad 0<\sigma_{1}<1,0<\sigma_{2}<1$ and $\sigma_{1}+\sigma_{2}=1$. Then, for any sufficiently large positive number $T>2$, we have

$$
\int_{2}^{T}\left|\zeta_{2}\left(s_{1}, s_{2}\right)\right|^{2} d t_{1}=\frac{\zeta(2)}{4 \pi\left|s_{2}-1\right|^{2}} T^{2}+O\left(t_{2}^{-\frac{1}{2}}\left(\log t_{2}\right) T^{\frac{3}{2}}\right)
$$

with $2 \leqslant t_{2} \leqslant \frac{T^{\frac{1}{3}}}{\log T}$.

Remark 1. Inserting $t_{2}=T^{\frac{1}{3}}(\log T)^{-1}$ into (1.9), the right-hand side of the formula (1.9) is estimated by $O\left(T^{\frac{4}{3}} \log ^{\frac{3}{2}} T\right)$, but if we can take $t_{2}=T$, we can estimate that $O(T \log T)$. The main term of this theorem is not $O\left(T \log ^{A} T\right)$ $(A>0)$, but $T^{2}$, since the analytic behaviour of the double zeta-function $\zeta_{2}\left(s_{1}, s_{2}\right)$ depends on both $s_{1}$ and $s_{2}$.

As an application of (1.9), we consider the evaluation of the double integral

$$
\int_{2}^{N} \int_{2}^{T}\left|\zeta_{2}\left(s_{1}, s_{2}\right)\right|^{2} d t_{1} d t_{2}
$$

and then we deduce the following.

Corollary 1. Let $0<\sigma_{1}<1,0<\sigma_{2}<1$ and $\sigma_{1}+\sigma_{2}=1$. Within the region $2 \leqslant N \leqslant \frac{T^{\frac{1}{3}}}{\log T}$, we obtain

$$
\lim _{T \rightarrow \infty} \frac{1}{T^{2}} \int_{2}^{N} \int_{2}^{T}\left|\zeta_{2}\left(s_{1}, s_{2}\right)\right|^{2} d t_{1} d t_{2}=\frac{\pi}{24 \sigma_{1}}\left(\frac{\pi}{2}-\operatorname{Tan}^{-1} \frac{2}{\sigma_{1}}\right)+O\left(\frac{1}{N}\right) .
$$

Hence, this observation may be regarded as an average order of magnitude for the double integral $\int_{2}^{N} \int_{2}^{T}\left|\zeta_{2}\left(s_{1}, s_{2}\right)\right|^{2} d t_{1} d t_{2}$, which is

$$
\frac{\pi}{24 \sigma_{1}}\left(\frac{\pi}{2}-\operatorname{Tan}^{-1} \frac{2}{\sigma_{1}}\right)
$$

for $0<\sigma_{1}<1,0<\sigma_{2}<1$ and $\sigma_{1}+\sigma_{2}=1$. 
Theorem 2. Suppose that $2 \leqslant t_{1} \leqslant T, 0<\sigma_{1}<1,0<\sigma_{2}<1, \frac{1}{2} \leqslant \sigma_{1}+\sigma_{2}<\frac{3}{2}$ and $\sigma_{1}+\sigma_{2} \neq 1$. Then, for any sufficiently large positive number $T>2$, we have

$$
\begin{aligned}
\int_{2}^{T}\left|\zeta_{2}\left(s_{1}, s_{2}\right)\right|^{2} d t_{1}= & (2 \pi)^{2 \sigma_{1}+2 \sigma_{2}-3} \frac{\zeta\left(4-2 \sigma_{1}-2 \sigma_{2}\right)}{\left(4-2 \sigma_{1}-2 \sigma_{2}\right)\left|s_{2}-1\right|^{2}} T^{4-2 \sigma_{1}-2 \sigma_{2}} \\
& +O\left(t_{2}^{-\frac{1}{2}} T^{\frac{5}{2}-\sigma_{1}-\sigma_{2}}\right)
\end{aligned}
$$

with $1<\sigma_{1}+\sigma_{2}<\frac{3}{2}$ and $2 \leqslant t_{2} \leqslant T^{1-\frac{2}{3}\left(\sigma_{1}+\sigma_{2}\right)}$,

$$
\begin{aligned}
\int_{2}^{T}\left|\zeta_{2}\left(s_{1}, s_{2}\right)\right|^{2} d t_{1}= & (2 \pi)^{2 \sigma_{1}+2 \sigma_{2}-3} \frac{\zeta\left(4-2 \sigma_{1}-2 \sigma_{2}\right)}{\left(4-2 \sigma_{1}-2 \sigma_{2}\right)\left|s_{2}-1\right|^{2}} T^{4-2 \sigma_{1}-2 \sigma_{2}} \\
& +O\left(t_{2}^{\frac{1}{2}-\sigma_{1}-\sigma_{2}} T^{\frac{5}{2}-\sigma_{1}-\sigma_{2}}\right)
\end{aligned}
$$

with $\frac{1}{2}<\sigma_{1}+\sigma_{2}<1$ and $2 \leqslant t_{2} \leqslant T^{\frac{3-2 \sigma_{1}-2 \sigma_{2}}{5-2 \sigma_{1}-2 \sigma_{2}}}$, and

$$
\begin{aligned}
\int_{2}^{T}\left|\zeta_{2}\left(s_{1}, s_{2}\right)\right|^{2} d t_{1}= & \frac{\zeta(3)}{12 \pi^{2}\left|s_{2}-1\right|^{2}} T^{3} \\
& +\left\{\begin{array}{cl}
O\left(T^{2}\right) & \text { if } \sqrt{\log T} \leqslant t_{2} \leqslant T^{\frac{1}{2}} \\
O\left(t_{2}^{-1} T^{2} \sqrt{\log T}\right) & \text { if } 2 \leqslant t_{2} \leqslant \sqrt{\log T}
\end{array}\right.
\end{aligned}
$$

with $\sigma_{1}+\sigma_{2}=\frac{1}{2}$.

In a similar manner to the proof of Theorem 2, we consider the integral $\int_{2}^{T}\left|\zeta_{2}\left(s_{1}, s_{2}\right)\right|^{2} d t_{1}$ for $\frac{3}{2}<\sigma_{1}+\sigma_{2}<2$, which may show the formula

$$
\int_{2}^{T}\left|\zeta_{2}\left(s_{1}, s_{2}\right)\right|^{2} d t_{1}=O\left(t_{2} T\right)
$$

but this does not provides an improvement on the formula (1.2). Similarly, in the case of $\sigma_{1}+\sigma_{2}=\frac{3}{2}$, we obtain the formula

$$
\int_{2}^{T}\left|\zeta_{2}\left(s_{1}, s_{2}\right)\right|^{2} d t_{1}=\frac{1}{\left|s_{2}-1\right|^{2}} T \log T+O\left(t_{2} T\right)
$$

for $2 \leqslant t_{2}<(\log T)^{\frac{1}{3}}$, which does not give an improvement upon the formula (1.3), since the error term of this formula depends on $t_{2}$.

Theorem 3. Suppose that $2 \leqslant t_{1} \leqslant T, \quad 0<\sigma_{1}<1, \quad 0<\sigma_{2}<1$ and $0<$ $\sigma_{1}+\sigma_{2}<\frac{1}{2}$. Then, for any sufficiently large positive number $T>2$, we have

$$
\begin{aligned}
& \int_{2}^{T}\left|\zeta_{2}\left(s_{1}, s_{2}\right)\right|^{2} d t_{1}=(2 \pi)^{2 \sigma_{1}+2 \sigma_{2}-3} \frac{\zeta\left(4-2 \sigma_{1}-2 \sigma_{2}\right)}{\left(4-2 \sigma_{1}-2 \sigma_{2}\right)\left|s_{2}-1\right|^{2}} T^{4-2 \sigma_{1}-2 \sigma_{2}} \\
& +\left\{\begin{array}{ccc}
O\left(t_{2}^{\frac{1}{2}-\sigma_{1}-\sigma_{2}} T^{\frac{5}{2}-\sigma_{1}-\sigma_{2}}\right) & \text { if } & T^{\frac{1-2 \sigma_{1}-2 \sigma_{2}}{3-2 \sigma_{1}-2 \sigma_{2}}} \leqslant t_{2} \leqslant T^{\frac{3-2 \sigma_{1}-2 \sigma_{2}}{5-2 \sigma_{1}-2 \sigma_{2}}} \\
O\left(t_{2}^{-1} T^{3-2 \sigma_{1}-2 \sigma_{2}}\right) & \text { if } & 2 \leqslant t_{2} \leqslant T^{\frac{1-2 \sigma_{1}-2 \sigma_{2}}{3-2 \sigma_{1}-2 \sigma_{2}}}
\end{array}\right.
\end{aligned}
$$


Remark 2. An average order of magnitude for the double zeta-function (1.1) derived from the asymptotic behaviour of the integrals of the double zeta-function $\zeta_{2}\left(s_{1}, s_{2}\right)$ with (1.9)-(1.13) holds when the ratio of the order of $t_{1}$ to that of $t_{2}$ is small. However, it is often difficult to determine their analytic behaviour for a general ratio of the order of $t_{1}$ to the order of $t_{2}$.

Sometimes it is more fruitful to study the integrals for the double zeta-function $\zeta_{2}\left(s_{1}, s_{2}\right)$ for $0<\sigma_{1}<1$ and $0<\sigma_{2}<1$.

Furthermore, the asymptotic behaviour of the $\Omega$ result of the double zetafunction (1.1) was derived by Kiuchi, Tanigawa, and Zhai [10], who showed that for $\sigma_{1}>0, \sigma_{2}>0, \sigma_{1}+\sigma_{2} \leqslant 1$ and

$$
t_{2} \ll t_{1}^{\frac{3-2 \sigma_{1}-2 \sigma_{2}}{7-2 \sigma_{1}-2 \sigma_{2}}-\varepsilon}
$$

then we have

$$
\zeta_{2}\left(\sigma_{1}+i t_{1}, \sigma_{2}+i t_{2}\right) \asymp \frac{t_{1}^{\frac{3}{2}-\sigma_{1}-\sigma_{2}}}{t_{2}}
$$

with $\sigma_{1}+\sigma_{2}<1$, and

$$
\zeta_{2}\left(\sigma_{1}+i t_{1}, \sigma_{2}+i t_{2}\right)=\Omega\left(\frac{t_{1}^{\frac{1}{2}} \log \log t_{1}}{t_{2}}\right)
$$

with $\sigma_{1}+\sigma_{2}=1$. For example, if we can take $t_{2} \ll t_{1}^{\frac{1}{6}-\varepsilon}$, then (1.16) implies that

$$
\zeta_{2}\left(\frac{1}{2}+i t_{1}, \frac{1}{2}+i t_{2}\right)=\Omega\left(t_{1}^{\frac{1}{3}+\varepsilon}\right) .
$$

In view of this, it is of some interest to try to determine the $\Omega$ result of the double zeta-function under the other region. From Theorems 1, 2, and 3, we are able to expand the region $\sigma_{1}>0, \sigma_{2}>0$ and $\sigma_{1}+\sigma_{2} \leqslant 1$ of their result into $0<\sigma_{1}<1$, $0<\sigma_{2}<1$ and $0<\sigma_{1}+\sigma_{2}<\frac{3}{2}$, and also improve a relation between $t_{1}$ and $t_{2}$ with the inequality (1.14). Thus we deduce the following $\Omega$ results.

Corollary 2. Suppose that $0<\sigma_{1}<1,0<\sigma_{2}<1$, and $2 \leqslant t_{1} \leqslant T$. Then we have

$$
\zeta_{2}\left(\sigma_{1}+i t_{1}, \sigma_{2}+i t_{2}\right)=\Omega\left(\frac{t_{1}^{\frac{3}{2}-\sigma_{1}-\sigma_{2}}}{t_{2}}\right)
$$

with $0<\sigma_{1}+\sigma_{2}<1$ and $2 \leqslant t_{2} \leqslant T^{\frac{3-2 \sigma_{1}-2 \sigma_{2}}{5-2 \sigma_{1}-2 \sigma_{2}}-\varepsilon}$, and

$$
\zeta_{2}\left(\sigma_{1}+i t_{1}, \sigma_{2}+i t_{2}\right)=\Omega\left(\frac{t_{1}^{\frac{3}{2}-\sigma_{1}-\sigma_{2}}}{t_{2}}\right)
$$

with $1 \leqslant \sigma_{1}+\sigma_{2}<\frac{3}{2}$ and $2 \leqslant t_{2} \leqslant T^{1-\frac{2}{3}\left(\sigma_{1}+\sigma_{2}\right)-\varepsilon}$, where $\varepsilon$ is any small positive constant. 
Remark 3. For the region $T^{\frac{3-2 \sigma_{1}-2 \sigma_{2}}{7-2 \sigma_{1}-2 \sigma_{2}}-\varepsilon} \ll t_{2} \leqslant T^{\frac{3-2 \sigma_{1}-2 \sigma_{2}}{5-2 \sigma_{1}-2 \sigma_{2}}-\varepsilon}$, the $\Omega$ result of (1.17) holds, hence this observation expands (1.15) into (1.17). For the case of $\sigma_{1}+\sigma_{2}=1$, the formula (1.16) holds for $t_{2} \ll t_{1}^{\frac{1}{5}-\varepsilon}$, but the formula (1.18) holds for $t_{2} \leqslant T^{\frac{1}{3}-\varepsilon}$. Furthermore, in the case where $1<\sigma_{1}+\sigma_{2}<\frac{3}{2}$, the formula (1.18) holds for $2 \leqslant t_{2} \leqslant T^{1-\frac{2}{3}\left(\sigma_{1}+\sigma_{2}\right)-\varepsilon}$. Therefore, this corollary implies an improvement upon the result of Kiuchi, Tanigawa and Zhai.

Remark 4. Now, we assume that $0<\sigma_{1}<1,0<\sigma_{2}<1, \sigma_{1}+\sigma_{2}=1,2<t_{1}<T$ and $2<t_{2}<T^{\frac{1}{2}}$. If the order of magnitude of the function $E\left(s_{1}, s_{2}\right)$ below is estimated as $O\left(t_{2}^{\varepsilon}\right)$ with $\varepsilon$ being any small positive constant by more elaborate analysis. Then, the formula (1.9) can be improved by

$$
\int_{2}^{T}\left|\zeta_{2}\left(s_{1}, s_{2}\right)\right|^{2} d t_{1}=\frac{\zeta(2)}{4 \pi\left|s_{2}-1\right|^{2}} T^{2}+O\left(t_{2}^{-1+\varepsilon} T^{\frac{3}{2}}\right)
$$

for any sufficiently large positive number $T>2$. Taking $t_{2}=T^{\frac{1}{2}-\varepsilon}$ into (1.19), we obtain the estimation

$$
\int_{2}^{T}\left|\zeta_{2}\left(s_{1}, s_{2}\right)\right|^{2} d t_{1}=O\left(T^{1+\varepsilon}\right) .
$$

Notations. When $g(x)$ is a positive function of $x$ for $x \geqslant x_{0}, f(x)=\Omega(g(x))$ means that $f(x)=o(g(x))$ does not hold as $x \rightarrow \infty$. In what follows, $\varepsilon$ denotes any arbitrarily small positive number, not necessarily the same ones at each occurrence.

\section{Some lemmas}

Let $a$ be any complex number. The generalized divisor function is defined by $\sigma_{a}(n)=\sum_{d \mid n} d^{a}$. We use a weak form of the approximate formula of Kiuchi, Tanigawa, and Zhai [10] to prove our theorems. Suppose that $0<\sigma_{1}<1$ and $0<\sigma_{2}<1$. They showed that

$$
\begin{aligned}
\zeta_{2}\left(s_{1}, s_{2}\right)= & \frac{\zeta\left(s_{1}+s_{2}-1\right)}{s_{2}-1}-\frac{1}{2} \zeta\left(s_{1}+s_{2}\right) \\
& +\chi\left(s_{2}\right) \sum_{n \leqslant \frac{\left|t_{2}\right|}{2 \pi}} \frac{\sigma_{1-s_{1}-s_{2}}(n)}{n^{1-s_{2}}}+O\left(\left|t_{2}\right|^{\max \left(0,1-\sigma_{1}-\sigma_{2}\right)+\varepsilon}\right)
\end{aligned}
$$

where

$$
\chi\left(s_{2}\right)=2(2 \pi)^{s_{2}-1} \sin \left(\frac{\pi}{2} s_{2}\right) \Gamma\left(1-s_{2}\right) .
$$

For our purpose, it is enough to quote the following weak form of (2.1):

$$
\zeta_{2}\left(s_{1}, s_{2}\right)=\frac{\zeta\left(s_{1}+s_{2}-1\right)}{s_{2}-1}-\frac{1}{2} \zeta\left(s_{1}+s_{2}\right)+E\left(s_{1}, s_{2}\right)
$$


with the error term $E\left(s_{1}, s_{2}\right)$. Using the partial summation formula, the estimate $\left|\chi\left(s_{2}\right)\right| \asymp\left|t_{2}\right|^{\frac{1}{2}-\sigma_{2}}$ and the partial sums of the generalized divisor function:

$$
\sum_{n \leqslant x} \sigma_{\alpha}(n)= \begin{cases}\frac{\zeta(\alpha+1)}{\alpha+1} x^{\alpha+1}+O\left(x^{\max (1, \alpha)}\right) & \text { if } \alpha>0, \\ x \log x+O(x) & \text { if } \alpha=0 \\ O(x) & \text { if } \alpha<0\end{cases}
$$

for any sufficiently large positive number $x>2$, we have the following.

Lemma 1. Let the notation be as above. We have

$$
E\left(s_{1}, s_{2}\right) \ll \begin{cases}\left|t_{2}\right|^{\frac{3}{2}-\sigma_{1}-\sigma_{2}} & \text { if } 0<\sigma_{1}+\sigma_{2}<1, \\ \left|t_{2}\right|^{\frac{1}{2}} \log \left|t_{2}\right| & \text { if } \sigma_{1}+\sigma_{2}=1, \\ \left|t_{2}\right|^{\frac{1}{2}} & \text { if } \sigma_{1}+\sigma_{2}>1 .\end{cases}
$$

Note that the error term in this lemma is independent of $t_{1}$. Here we choosed $\varepsilon$ in $(2.1)$ as $0<\varepsilon<1 / 2$. This error term $E\left(s_{1}, s_{2}\right)$ is obviously independent of $t_{1}$. To prove our theorem, we shall establish the mean square formula for $\zeta_{2}\left(s_{1}, s_{2}\right)$ by using (2.2), (2.3) and Schwarz's inequality, thus we have the following.

Lemma 2. For $0<\sigma_{1}<1,0<\sigma_{2}<1$, and any sufficiently large number $T>2$, we have

$$
\int_{2}^{T}\left|\zeta_{2}\left(s_{1}, s_{2}\right)\right|^{2} d t_{1}=I_{1}+I_{2}+I_{3}+O\left(I_{1}^{\frac{1}{2}} I_{2}^{\frac{1}{2}}+I_{2}^{\frac{1}{2}} I_{3}^{\frac{1}{2}}+I_{3}^{\frac{1}{2}} I_{1}^{\frac{1}{2}}\right),
$$

where

$$
\begin{aligned}
I_{1} & =\frac{1}{\left|s_{2}-1\right|^{2}} \int_{2}^{T}\left|\zeta\left(s_{1}+s_{2}-1\right)\right|^{2} d t_{1}, \\
I_{2} & =\frac{1}{4} \int_{2}^{T}\left|\zeta\left(s_{1}+s_{2}\right)\right|^{2} d t_{1},
\end{aligned}
$$

and

$$
I_{3}=\int_{2}^{T}\left|E\left(s_{1}, s_{2}\right)\right|^{2} d t_{1} .
$$

To deal with the integrals $I_{j}(j=1,2,3)$, we shall use the mean square formula of the Riemann zeta-function.

Lemma 3. For any sufficiently large positive number $T>2$, we have

$$
\int_{2}^{T}|\zeta(\sigma+i t)|^{2} d t=(2 \pi)^{2 \sigma-1} \frac{\zeta(2-2 \sigma)}{2-2 \sigma} T^{2-2 \sigma}+\zeta(2 \sigma) T+O\left(T^{1-\sigma}\right)
$$


with $0<\sigma<\frac{1}{2}$,

$$
\int_{2}^{T}|\zeta(i t)|^{2} d t=\frac{\zeta(2)}{4 \pi} T^{2}+\frac{1}{2 \pi} R(T) T+O(T)
$$

with $\sigma=0$, and

$$
\int_{2}^{T}|\zeta(\sigma+i t)|^{2} d t=(2 \pi)^{2 \sigma-1} \frac{\zeta(2-2 \sigma)}{2-2 \sigma} T^{2-2 \sigma}+O\left(T^{1-2 \sigma}\right)
$$

with $-1<\sigma<0$, where $R(T)$ is given by (1.7).

Proof. Since the functional equation $\zeta(\sigma+i t)=\chi(\sigma+i t) \zeta(1-\sigma-i t)$, the equality $|\zeta(1-\sigma-i t)|=|\zeta(1-\sigma+i t)|$ and the formula (see (1.25) in [6])

$$
|\chi(\sigma+i t)|^{2}=\left(\frac{t}{2 \pi}\right)^{1-2 \sigma}+O\left(t^{-2 \sigma}\right) \quad\left(t \geqslant t_{0}>0\right)
$$

we can integrate by parts and use (1.6) to obtain

$$
\begin{aligned}
\int_{2}^{T}|\zeta(\sigma+i t)|^{2} d t= & \int_{2}^{T}|\chi(\sigma+i t)|^{2}|\zeta(1-\sigma+i t)|^{2} d t \\
= & \left(\frac{1}{2 \pi}\right)^{1-2 \sigma} \int_{2}^{T} t^{1-2 \sigma}|\zeta(1-\sigma+i t)|^{2} d t \\
& +O\left(\int_{2}^{T} t^{-2 \sigma}|\zeta(1-\sigma+i t)|^{2} d t\right) \\
= & (2 \pi)^{2 \sigma-1} \frac{\zeta(2-2 \sigma)}{2-2 \sigma} T^{2-2 \sigma}+\zeta(2 \sigma) T+O\left(T^{1-\sigma}\right)
\end{aligned}
$$

with $E_{1-\sigma}(T)=O\left(T^{\sigma}\right)$ for $0<\sigma<\frac{1}{2}$. Similarly in the case of $\sigma=0$, we have, by integrating by parts and using (1.7), (1.8),

$$
\begin{aligned}
\int_{2}^{T}|\zeta(i t)|^{2} d t & =\int_{2}^{T}|\chi(i t)|^{2}|\zeta(1+i t)|^{2} d t \\
& =\frac{1}{2 \pi} \int_{2}^{T} t|\zeta(1+i t)|^{2} d t+O\left(\int_{2}^{T}|\zeta(1+i t)|^{2} d t\right) \\
& =\frac{\zeta(2)}{4 \pi} T^{2}+\frac{1}{2 \pi} R(T) T+O(T) .
\end{aligned}
$$


In a similar manner, we have,

$$
\begin{aligned}
\int_{2}^{T}|\zeta(\sigma+i t)|^{2} d t= & \int_{2}^{T}|\chi(\sigma+i t)|^{2}|\zeta(1-\sigma+i t)|^{2} d t \\
= & \left(\frac{1}{2 \pi}\right)^{1-2 \sigma} \int_{2}^{T} t^{1-2 \sigma}|\zeta(1-\sigma+i t)|^{2} d t \\
& +O\left(\int_{2}^{T} t^{-2 \sigma}|\zeta(1-\sigma+i t)|^{2} d t\right) \\
= & (2 \pi)^{2 \sigma-1} \frac{\zeta(2-2 \sigma)}{2-2 \sigma} T^{2-2 \sigma}+O\left(T^{1-2 \sigma}\right)
\end{aligned}
$$

for $-1<\sigma<0$, which gives the formula (2.10).

\section{Proofs of Theorem 1 and Corollary 1}

We evaluate the integral $\int_{2}^{T}\left|\zeta_{2}\left(s_{1}, s_{2}\right)\right|^{2} d t_{1}$ under the condition $0<\sigma_{1}<1$, $0<\sigma_{2}<1, \sigma_{1}+\sigma_{2}=1$, and $2 \leqslant t_{2} \leqslant \frac{T^{\frac{1}{3}}}{\log T}$. From (1.7), we have

$$
I_{2}=\frac{1}{4}\left\{\int_{2}^{T+t_{2}}|\zeta(1+i t)|^{2} d t-\int_{2}^{2+t_{2}}|\zeta(1+i t)|^{2} d t\right\}=O\left(T+t_{2}\right) .
$$

Similarly, we have

$$
I_{1}=\frac{1}{\left|s_{2}-1\right|^{2}}\left\{\int_{2}^{T+t_{2}}|\zeta(i t)|^{2} d t-\int_{2}^{2+t_{2}}|\zeta(i t)|^{2} d t\right\} .
$$

Inserting (2.9) into (3.2), we obtain

$$
\begin{aligned}
I_{1}= & \frac{\zeta(2)}{4 \pi\left|s_{2}-1\right|^{2}}\left\{\left(T+t_{2}\right)^{2}-\left(t_{2}+2\right)^{2}\right\} \\
& +\frac{1}{2 \pi\left|s_{2}-1\right|^{2}}\left\{\left(T+t_{2}\right) R\left(T+t_{2}\right)-\left(t_{2}+2\right) R\left(t_{2}+2\right)\right\}+O\left(\frac{T+t_{2}}{\left|s_{2}-1\right|^{2}}\right) \\
= & \frac{\zeta(2)}{4 \pi\left|s_{2}-1\right|^{2}}\left(T+t_{2}\right)^{2}+O\left(\frac{T(\log T)^{2 / 3}(\log \log T)^{1 / 3}}{t_{2}^{2}}\right)+O\left(\frac{T}{t_{2}^{2}}\right)
\end{aligned}
$$

We have

$$
I_{3}=O\left(\left(t_{2} \log ^{2} t_{2}\right) T\right)
$$

Substituting (3.1), (3.3) and (3.4) into (2.4), we observe that all error terms on the right-hand side of $(2.4)$ are absorbed into $O\left(t_{2}^{-\frac{1}{2}}\left(\log t_{2}\right) T^{\frac{3}{2}}\right)$, completing the proof of (1.9). 
Next, as an application of (1.9), we evaluate the double integral for the double zeta-function $\zeta_{2}\left(s_{1}, s_{2}\right): \int_{2}^{N} \int_{2}^{T}\left|\zeta_{2}\left(s_{1}, s_{2}\right)\right|^{2} d t_{1} d t_{2}$. From (1.9), we have

$$
\begin{aligned}
& \int_{2}^{N} \int_{2}^{T}\left|\zeta_{2}\left(s_{1}, s_{2}\right)\right|^{2} d t_{1} d t_{2} \\
& \quad=\frac{\zeta(2)}{4 \pi}\left(\int_{2}^{N} \frac{1}{\left|s_{2}-1\right|^{2}} d t_{2}\right) T^{2}+O\left(T^{\frac{3}{2}} \int_{2}^{N} t_{2}^{-\frac{1}{2}} \log t_{2} d t_{2}\right)
\end{aligned}
$$

for $2 \leqslant N \leqslant \frac{T^{\frac{1}{3}}}{\log T}$. It follows that for $0<\sigma_{2}<1$

$$
\int_{2}^{N} \frac{1}{\left|s_{2}-1\right|^{2}} d t_{2}=\frac{1}{1-\sigma_{2}}\left(\frac{\pi}{2}-\operatorname{Tan}^{-1} \frac{2}{1-\sigma_{2}}\right)+O\left(\frac{1}{N}\right)
$$

and

$$
\int_{2}^{N} t_{2}^{-\frac{1}{2}} \log t_{2} d t_{2}=O\left(N^{\frac{1}{2}} \log N\right) .
$$

Then, we easily see that

$$
\begin{aligned}
\int_{2}^{N} \int_{2}^{T}\left|\zeta_{2}\left(s_{1}, s_{2}\right)\right|^{2} d t_{1} d t_{2}= & \frac{\pi}{24\left(1-\sigma_{2}\right)}\left(\frac{\pi}{2}-\operatorname{Tan}^{-1} \frac{2}{1-\sigma_{2}}\right) T^{2} \\
& +O\left(T^{\frac{3}{2}} N^{\frac{1}{2}} \log N\right)+O\left(\frac{T^{2}}{N}\right)
\end{aligned}
$$

Hence, for $0<\sigma_{1}<1,0<\sigma_{2}<1, \sigma_{1}+\sigma_{2}=1$ and $2 \leqslant N \leqslant \frac{T^{\frac{1}{3}}}{\log T}$, we obtain, as $T \rightarrow \infty$

$$
\frac{1}{T^{2}} \int_{2}^{N} \int_{2}^{T}\left|\zeta_{2}\left(s_{1}, s_{2}\right)\right|^{2} d t_{1} d t_{2} \rightarrow \frac{\pi}{24 \sigma_{1}}\left(\frac{\pi}{2}-\operatorname{Tan}^{-1} \frac{2}{\sigma_{1}}\right)+O\left(\frac{1}{N}\right) .
$$

Therefore, we obtain the assertion of Corollary 1 .

\section{Proof of Theorem 2}

Throughout this section, we assume that $0<\sigma_{1}<1,0<\sigma_{2}<1, \frac{1}{2} \leqslant \sigma_{1}+\sigma_{2}<\frac{3}{2}$, $\sigma_{1}+\sigma_{2} \neq 1$ and $2 \leqslant t_{2} \leqslant T^{\frac{2}{3}}$. As in the proof of Theorem 1 , we shall first evaluate the integral $\int_{2}^{T}\left|\zeta_{2}\left(s_{1}, s_{2}\right)\right|^{2} d t_{1}$ for $1<\sigma_{1}+\sigma_{2}<\frac{3}{2}$. Using (1.4) and (2.6), we obtain

$$
I_{2}=\frac{1}{4} \int_{2}^{T}\left|\zeta\left(s_{1}+s_{2}\right)\right|^{2} d t_{1}=\frac{1}{4} \zeta\left(2\left(\sigma_{1}+\sigma_{2}\right)\right) T+O\left(t_{2}\right) .
$$

From (2.3) and (2.7), we have

$$
I_{3}=O\left(t_{2} T\right)
$$


By (2.5) and (2.8), we have

$$
\begin{aligned}
I_{1}= & \frac{1}{\left|s_{2}-1\right|^{2}} \int_{2}^{T}\left|\zeta\left(\sigma_{1}+\sigma_{2}-1+i\left(t_{1}+t_{2}\right)\right)\right|^{2} d t_{1} \\
= & \frac{(2 \pi)^{2 \sigma_{1}+2 \sigma_{2}-3}}{\left|s_{2}-1\right|^{2}} \frac{\zeta\left(4-2 \sigma_{1}-2 \sigma_{2}\right)}{4-2 \sigma_{1}-2 \sigma_{2}} T^{4-2 \sigma_{1}-2 \sigma_{2}} \\
& +O\left(t_{2}^{-1} T^{3-2 \sigma_{1}-2 \sigma_{2}}\right)+O\left(t_{2}^{-2} T\right) .
\end{aligned}
$$

Now we assume that $2 \leqslant t_{2} \leqslant T^{1-\frac{2}{3}\left(\sigma_{1}+\sigma_{2}\right)}$. Substituting (4.1), (4.2) and (4.3) into (2.4), we observe that all error terms on the right-hand side of (2.4) are absorbed into $O\left(t_{2}^{-\frac{1}{2}} T^{\frac{5}{2}-\sigma_{1}-\sigma_{2}}\right)$. Hence, we derive the formula (1.10).

Similarly, in the case of $\frac{1}{2}<\sigma_{1}+\sigma_{2}<1$, we obtain

$$
I_{2}=\frac{1}{4} \zeta\left(2\left(\sigma_{1}+\sigma_{2}\right)\right) T+A\left(\sigma_{1}+\sigma_{2}\right) T^{2-2 \sigma_{1}-2 \sigma_{2}}+O\left(t_{2}\right)
$$

by using (1.6) and (2.6). From (2.3) and (2.7), we have

$$
I_{3}=O\left(t_{2}^{3-2 \sigma_{1}-2 \sigma_{2}} T\right) \text {. }
$$

From (2.5) and (2.10), we have

$$
I_{1}=\frac{(2 \pi)^{2 \sigma_{1}+2 \sigma_{2}-3}}{\left|s_{2}-1\right|^{2}} \frac{\zeta\left(4-2 \sigma_{1}-2 \sigma_{2}\right)}{4-2 \sigma_{1}-2 \sigma_{2}} T^{4-2 \sigma_{1}-2 \sigma_{2}}+O\left(t_{2}^{-1} T^{3-2 \sigma_{1}-2 \sigma_{2}}\right) .
$$

Now we assume that $2 \leqslant t_{2} \leqslant T^{\frac{3-2 \sigma_{1}-2 \sigma_{2}}{5-2 \sigma_{1}-2 \sigma_{2}}}$. Substituting (4.4), (4.5) and (4.6) into (2.4), we observe that all error terms on the right-hand side of (2.4) are absorbed into $O\left(t_{2}^{\frac{1}{2}-\sigma_{1}-\sigma_{2}} T^{\frac{5}{2}-\sigma_{1}-\sigma_{2}}\right)$. Hence, we derive the formula (1.11).

Similarly, in the case of $\sigma_{1}+\sigma_{2}=\frac{1}{2}$, we obtain, by (1.5) and (2.6)

$$
I_{2}=T \log \frac{T}{2 \pi}+(2 \gamma-1) T+O\left(t_{2} \log T+T^{\frac{1}{2}}\right) .
$$

From (2.3) and (2.7), we have

$$
I_{3}=O\left(t_{2}^{2} T\right)
$$

From (2.5) and (2.10), we have

$$
I_{1}=\frac{\zeta(3)}{12 \pi^{2}\left|s_{2}-1\right|^{2}} T^{3}+O\left(t_{2}^{-1} T^{2}\right) .
$$

Now we assume that $2 \leqslant t_{2} \leqslant T^{\frac{1}{2}}$. Substituting (4.7), (4.8) and (4.9) into (2.4), we observe that all error terms on the right-hand side of (2.4) are absorbed into $O\left(t_{2}^{-1} T^{2} \sqrt{\log T}\right)$ if $2 \leqslant t_{2} \leqslant \sqrt{\log T}$, or into $O\left(T^{2}\right)$ if $\sqrt{\log T} \leqslant t_{2} \leqslant T^{\frac{1}{2}}$. Hence, we obtain the formula (1.12). 


\section{Proof of Theorem 3}

Let $0<\sigma_{1}<1,0<\sigma_{2}<1$ and $2 \leqslant t_{2} \leqslant T^{\frac{2}{3}}$. As in the proof of Theorem 1 , we shall evaluate the integral $\int_{2}^{T}\left|\zeta_{2}\left(s_{1}, s_{2}\right)\right|^{2} d t_{1}$ for $0<\sigma_{1}+\sigma_{2}<\frac{1}{2}$. From (2.6) and (2.8), we have

$$
I_{2}=O\left(T^{2-2 \sigma_{1}-2 \sigma_{2}}\right)
$$

By (2.3) and (2.7), we have

$$
I_{3}=O\left(t_{2}^{3-2 \sigma_{1}-2 \sigma_{2}} T\right)
$$

By (2.5) and (2.10), we obtain

$$
I_{1}=\frac{(2 \pi)^{2 \sigma_{1}+2 \sigma_{2}-3}}{\left|s_{2}-1\right|^{2}} \frac{\zeta\left(4-2 \sigma_{1}-2 \sigma_{2}\right)}{4-2 \sigma_{1}-2 \sigma_{2}} T^{4-2 \sigma_{1}-2 \sigma_{2}}+O\left(t_{2}^{-1} T^{3-2 \sigma_{1}-2 \sigma_{2}}\right) .
$$

Now we assume that $2 \leqslant t_{2} \leqslant T^{\frac{3-2 \sigma_{1}-2 \sigma_{2}}{5-2 \sigma_{1}-2 \sigma_{2}}}$. Substituting (5.1), (5.2) and (5.3) into (2.4), we observe that all error terms on the right-hand side of (2.4) are absorbed into

$$
O\left(t_{2}^{\frac{1}{2}-\sigma_{1}-\sigma_{2}} T^{\frac{5}{2}-\sigma_{1}-\sigma_{2}}\right) \quad \text { if } T^{\frac{1-2 \sigma_{1}-2 \sigma_{2}}{3-2 \sigma_{1}-2 \sigma_{2}}} \leqslant t_{2} \leqslant T^{\frac{3-2 \sigma_{1}-2 \sigma_{2}}{5-2 \sigma_{1}-2 \sigma_{2}}}
$$

or into

$$
O\left(t_{2}^{-1} T^{3-2 \sigma_{1}-2 \sigma_{2}}\right) \quad \text { if } 2 \leqslant t_{2} \leqslant T^{\frac{1-2 \sigma_{1}-2 \sigma_{2}}{3-2 \sigma_{1}-2 \sigma_{2}}} .
$$

Therefore, we obtain the formula (1.13).

\section{References}

[1] S. Akiyama, S. Egami and Y. Tanigawa, An analytic continuation of multiple zeta-functions and their values at non-positive integers, Acta Arith. 98 (2001), $107-116$.

[2] F.V. Atkinson, The mean-value of the Riemann zeta-function, Acta Math. 81 (1949), 353-376.

[3] R. Balasubramanian, A. Ivić and K. Ramachandra, The mean square of the Riemann zeta-function on the line $\sigma=1$, Enseign. Math. (2) 38 (1992), $13-25$.

[4] S. Ikeda, K. Matsuoka and Y. Nagata, On certain mean values of the double zeta-function, Nagoya Math. J. 217 (2015), 161-190.

[5] H. Ishikawa and K. Matsumoto, On the estimation of the order of Euler-Zagier multiple zeta-functions, Illinois J. Math. 47 (2003), 1151-1166.

[6] A. Ivić, The Riemann Zeta-Function, John Wiley and Sons, New York, 1985 (2nd ed. Dover, 2003).

[7] A. Ivić, Mean Values of the Riemann Zeta Function, Lecture Note Ser.82. Tata Institute of Fundamental Research, Bombay, Berlin-Heidelberg-New York; Springer 1991. 
[8] A. Ivić and K. Matsumoto, On the error term in the mean square formula for the Riemann zeta-function in the critical strip, Monatsh. Math. 121 (1996), $213-229$.

[9] I. Kiuchi and Y. Tanigawa, Bounds for double zeta-functions, Ann. Sc. Norm. Sup. Pisa, Cl. Sci. Ser. V 5 (2006), 445-464.

[10] I. Kiuchi, Y. Tanigawa and W. Zhai, Analytic properties of double zetafunctions, Indag. Math. 21 (2011), 16-29.

[11] K. Matsumoto, On the analytic continuation of various multiple zetafunctions, in: "Number Theory for the Millennium, Proc. Millennial Conf. Number Theory", Vol. II, M. A. Bennett et al. (eds.), A.K. Peters 2002, 417440.

[12] K. Matsumoto, Functional equations for double zeta-functions, Math. Proc. Cambridge Philos. Soc. 136 (2004), 1-7.

[13] K. Matsumoto and H. Tsumura, Mean value theorems for the double zetafunction, J. Math. Soc. Japan. 67 (2015), 383-406.

[14] E.C. Titchmarsh, The Theory of the Riemann Zeta-Function, Second Edition, Edited and with a preface by D. R. Heath-Brown, The Clarendon Press, Oxford University Press, New York, 1986.

[15] J.Q. Zhao, Analytic continuation of multiple zeta function, Proc. Amer. Math. Soc. 128 (2000), 1275-1283.

Address: Isao Kiuchi and Makoto Minamide: Department of Mathematical Sciences, Faculty of Science, Yamaguchi University, Yoshida 1677-1, Yamaguchi 753-8512, Japan.

E-mail: kiuchi@yamaguchi-u.ac.jp, minamide@yamaguchi-u.ac.jp

Received: 14 April 2015; revised: 19 November 2015 